\title{
Influence of glass composition on the luminescence mechanisms of CdSe quantum dot-doped glasses
}

\author{
Wenke Li ${ }^{1,2}$ Xiujian Zhao, ${ }^{1}$ Chao Liu,${ }^{1 *}$ François-Xavier Coudert ${ }^{2 *}$ \\ ${ }^{1}$ State Key Laboratory of Silicate Materials for Architectures, Wuhan University of \\ Technology, Hubei, 430070, China \\ ${ }^{2}$ Chimie ParisTech, PSL University, CNRS, Institut de Recherche de Chimie Paris,
} 75005 Paris, France

\begin{abstract}
:
In this work, we characterized the electronic structure of CdSe quantum dots embedded in a series of $x \mathrm{Na}_{2} \mathrm{O},(1-x) \mathrm{SiO}_{2}$ glass matrices $(x=0,0.25,0.33$ and 0.5$)$. We analyzed the impact of the glass matrix composition on both the atomic structure of the quantum dot (QD) and the QD/glass interface, as well as the luminescence mechanisms, using density functional theory (DFT) calculations. The increase of $\mathrm{Na}_{2} \mathrm{O}$ content in the glass matrices was found to promote the formation of $\mathrm{Cd}-\mathrm{O}$ and $\mathrm{Se}-\mathrm{Na}$ interfacial bonds, and disrupting the $\mathrm{Cd}-\mathrm{Se}$ bonds network. In particular, we show that the glass composition directly affects the nature of the highest occupied molecular orbitals (HOMO). According to the atomic structure, the band gap distribution and the density of states calculation, we find that there is significant reconstruction of the QD, and that the picture sometimes proposed of a "pristine QD" surrounded by glass is not realistic. The introduction of CdSe QD significantly decreased the bandgap of the glass compared to pristine glasses, and the interfacial bonds greatly contributed to the frontier orbitals without forming midgap states. We propose a new energy diagram, quite different from the traditional model, to explain the luminescence of CdSe quantum dotdoped glasses, originating from the intrinsic emission of this hybrid system \{QD + glass $\}$. These results improve our understanding of the luminescence of CdSe quantum dot-doped glasses, explaining the reason for the poor quantum efficiency and broad emission linewidth compared with their colloidal counterparts.
\end{abstract}




\section{Introduction}

Quantum dots (QDs) exhibit remarkable electronic and optical properties induced by quantum confinement. Colloidal quantum dots can be produced at low cost with high quantum efficiency and narrow size distribution, which makes them promising for photovoltaics $^{1}$, lighting ${ }^{2}$ and labeling ${ }^{3}$ applications. Incorporation of QDs into glasses can prevent the agglomeration, and enhance the chemical and thermal abilities, making them appealing for nonlinear optical devices and LEDs. ${ }^{4-5}$

Due to the high surface-to-volume ratio of quantum dots, the main obstacle for the success of their applications is to attain a precise control of the emission properties, which mainly depends on the surface chemistry of the nanocrystals, and the interface with the surrounding medium. Surface defects are ubiquitous to QDs, and a deep understanding of their atomic origin is necessary to tune the emission and design defectfree QDs. In spite of a wealth of experimental data reported for CdSe QDs, the question of exactly how surface defects influence the photophysical properties of QDs is still open. It is experimentally challenging to resolve spectral features originating from QDligand interactions. Density functional theory (DFT) are able to address these issues, gaining comparable gaps between the highest occupied (HOMO) and the lowest unoccupied molecular orbitals (LUMO), IR and Raman spectra to experimental findings. ${ }^{6}$

In the literature, $\mathrm{Cd}_{33} \mathrm{Se}_{33}$ QDs are the most commonly used representative models of quantum dots for computational studies, as they are believed to have the highest degree of stability, exhibiting basic optical features similar to larger size QDs. Kilina ${ }^{7}$ et al. found that the electron trap states were very sensitive to ligand position and the solvent polarity. Sargent et al. ${ }^{8}$ demonstrated that surface vacancies can improve the fluorescence yield compared to vacancy-free surfaces. Beratan et al. ${ }^{9}$ performed comparative studies of the structural and electronic properties of pristine and $\mathrm{NH}_{3}{ }^{-}$, $\mathrm{SCH}_{3}{ }^{-}$, and $\mathrm{OPH}_{2}{ }^{-}$capped $\mathrm{Cd}_{33} \mathrm{Se}_{33}$ QDs, showing that an increase in capping ligand concentration causes noticeable changes in the capped QD electronic structure, leading to different surface states and HOMO-LUMO gaps. Pudzder ${ }^{10}$ et al. reported significant reconstruction of the nanoparticle surface while the wurtzite core was maintained, leading to the opening of an optical gap without the aid of passivating ligands, thus "self-healing" the surface electronic structure.

All those theoretical studies helped understand the structural origin of defect emission and effective surface passivation of colloidal QDs. However, relatively little research effort has been devoted to the computational study of CdSe quantum-dots embedded in glasses compared to their colloidal counterparts. The quantum efficiency of the quantum dots embedded in glass matrices is extremely low, which is usually attributed to their surface passivation and broad size distribution. Recent work showed that the size distribution can be controlled by tuning the heat-treatment and the concentration of dopants. ${ }^{11}$ However, surface control techniques used in colloidal QDs, such as postsynthetic passivation by surfactants or growth of an iso-structural shell is difficult to 
complete, when the QDs are surrounded by dense and amorphous glass matrices.

Hence, defect emission, rather than intrinsic emission, is most frequently observed in the CdSe quantum-dot-doped glasses. It is of predominant importance to fully comprehend the structural origin of defect emission and the impact of QD/glass interface on the electronic structure. The atomic structure of the CdSe quantum-dotdoped glasses is exceedingly difficult to characterize due to the typical low density of QDs in the glass and the instability of glass matrices under electron beam. In previous work, ${ }^{12}$ we used network modifiers as well as non-bridging oxygen atoms to be capped with $\mathrm{Cd}_{33} \mathrm{Se}_{33}$ clusters, in order to explore the impact of these additional surface atoms on the morphology and electronic structure on the CdSe QD. However, this simplistic model was far from being a realistic environment of the quantum dot in glass matrices. In later work, we created a more detailed atomic structure by using a combination of classical and ab initio molecular dynamics, demonstrating the complex interfacial chemical environment between the CdSe quantum dot and a surrounding glass matrix. ${ }^{13}$

In the present work, we used DFT-based methods to determine the structure of CdSe quantum-dot-doped glasses with varying composition, and calculate their electronic structure to probe the impact of the glass matrices on the optical properties of the CdSe quantum dot. Besides, the sodium ion was found to sharply decrease the HOMOLUMO gap in previous DFT calculations ${ }^{12}$. In their role of glass modifiers, sodium ions can alter the structure of silicate glasses, and it was experimentally observed that there is a disappearance of the visible absorption of Se-Se color centers upon increasing concentration of $\mathrm{Na}_{2} \mathrm{O}$ in a silicate glass doped with $\mathrm{ZnSe} .{ }^{14}$ The Se-Se color centers are considered to be the nucleation sites of the CdSe QD. Therefore in this study we changed the amount of $\mathrm{Na}_{2} \mathrm{O}$ in the glass $\left(\mathrm{Na}_{2} \mathrm{O}\right)_{x}\left(\mathrm{SiO}_{2}\right)_{1-x}(x=0,0.25,0.33,0.5$ in molar fraction) to explore the compositional dependence of the atomic and electronic structure of CdSe quantum dot in glass matrices. The results demonstrate that an increase in the amount of $\mathrm{Na}_{2} \mathrm{O}$ contributes to the formation of $\mathrm{Cd}-\mathrm{O}$ and $\mathrm{Se}-\mathrm{Na}$ bonds, with breaking of $\mathrm{Si}-\mathrm{O}, \mathrm{O}-\mathrm{Na}$ and $\mathrm{Cd}-\mathrm{Se}$ bonds. The density of states (DOS) and projected density of states (PDOS) were also calculated. Although with the same composition, the dominant luminescence mechanisms in different configurations are also different. The top of the valence band and the bottom of the conduction band are decided by the hybrid QD in most compositions. However, in most configurations of CdSe quantum-dot-doped glass with composition $0.33 \mathrm{Na}_{2} \mathrm{O}-0.67 \mathrm{SiO}_{2}$ exhibit totally different luminescence mechanisms that the top of the valence band is determined by the hybrid glasses.

Most importantly, based on the analysis of atomic structure, HOMO-LUMO gap distribution and DOS, the intrinsic emission from pristine QD is found to be negligible due to the little possibility of QD without any interaction with glass matrix. The photoluminescence of CdSe quantum dots doped glass is originated from the intrinsic emission of these complicated hybrid system rather than the intrinsic or defect emission of pristine QD, which is a common model proposed by experimentalists. These results 
provide a better understanding of the electronic structure and luminescence mechanisms of these complex systems, giving guidance for future compositional design of highly luminescent glass containing quantum dots.

\section{Computational Methods}

Table 1 Composition of the different $x \mathrm{Na}_{2} \mathrm{O}-(1-x) \mathrm{SiO}_{2}$ (in molar fraction) glasses investigated in this study and their respective densities. For each system, we list the number of $\mathrm{O}, \mathrm{Si}, \mathrm{Na}, \mathrm{Cd}$ and $\mathrm{Se}$ atoms in the simulation cell of the CdSe quantum dotdoped glass.

\begin{tabular}{ccccccc}
\hline \multicolumn{2}{c}{ Pristine Glass } & \multicolumn{5}{c}{ CdSe quantum dot-doped glasses } \\
\hline$\left(\mathrm{Na}_{2} \mathrm{O}\right)_{x}\left(\mathrm{SiO}_{2}\right)_{1-x}$ & $\begin{array}{c}\text { Density } \\
\left(\mathrm{g} / \mathrm{cm}^{3}\right)\end{array}$ & $\mathrm{O}$ & $\mathrm{Si}$ & $\mathrm{Na}$ & $\mathrm{Cd}$ & $\mathrm{Se}$ \\
$\boldsymbol{x}=\mathbf{0}$ & 2.20 & 224 & 112 & -- & 33 & 33 \\
$\boldsymbol{x}=\mathbf{0 . 2 5}$ & 2.43 & 210 & 90 & 60 & 33 & 33 \\
$\boldsymbol{x}=\mathbf{0 . 3 3}$ & 2.49 & 195 & 78 & 78 & 33 & 33 \\
$x=0.5$ & 2.56 & 186 & 62 & 124 & 33 & 33 \\
\hline
\end{tabular}

We generated configurations of pristine sodium silicate glasses, and from those pristine glasses, created CdSe quantum dot-doped silicate glasses, using a combination of classical force field-based molecular dynamics (MD) and ab initio molecular dynamics (AIMD). The protocol followed here is adapted from that developed and validated in our previous work. ${ }^{13}$ The pristine QD was cut from the bulk structure and then relaxed to the lowest energy configuration. ${ }^{12}$ The pristine QD was directly introduced into the pristine glass matrix, which was the initial configuration for classical molecular dynamics. It was equilibrated at $1000 \mathrm{~K}$ and then cooled gradually from $1000 \mathrm{~K}$ to 500 $\mathrm{K}$ while keeping CdSe frozen, followed by quenching from $500 \mathrm{~K}$ to $300 \mathrm{~K}$ with all atoms allowed to move. Then the structures were further equilibrated at $300 \mathrm{~K}$. The resulting configuration was used as a starting configuration for AIMD, quenched from $500 \mathrm{~K}$ to $300 \mathrm{~K}$ and we obtained the hybrid system-CdSe quantum dot-doped glasses.

For each system, 500 configurations were selected at equal interval from a $10 \mathrm{ps}$ production run of $a b$ initio molecular dynamics simulations. Among those, 50 configurations were picked to perform the calculation and analysis of the electronic structure of CdSe quantum dot-doped glasses. We used the nonlocal PBE0-TC-LRC exchange-correlation functional with a cutoff radius of $2.0 \AA .{ }^{15}$ From previous work, the electronic structure calculations based on the generalized gradient approximation (GGA) or local density approximation (LDA) exchange-correlation functionals severely underestimated the HOMO-LUMO gap and long range Coulombic interactions. ${ }^{16-18}$ The inclusion of the Hartree-Fock exchanges was found in literature, to provide a more accurate description ${ }^{18-19}$ of the band gap. Moreover, the computational cost of nonlocal functional calculations can be reduced using the auxiliary density matrix method (ADMM). ${ }^{20}$ 


\section{Results and Discussion}

(a)

(c)
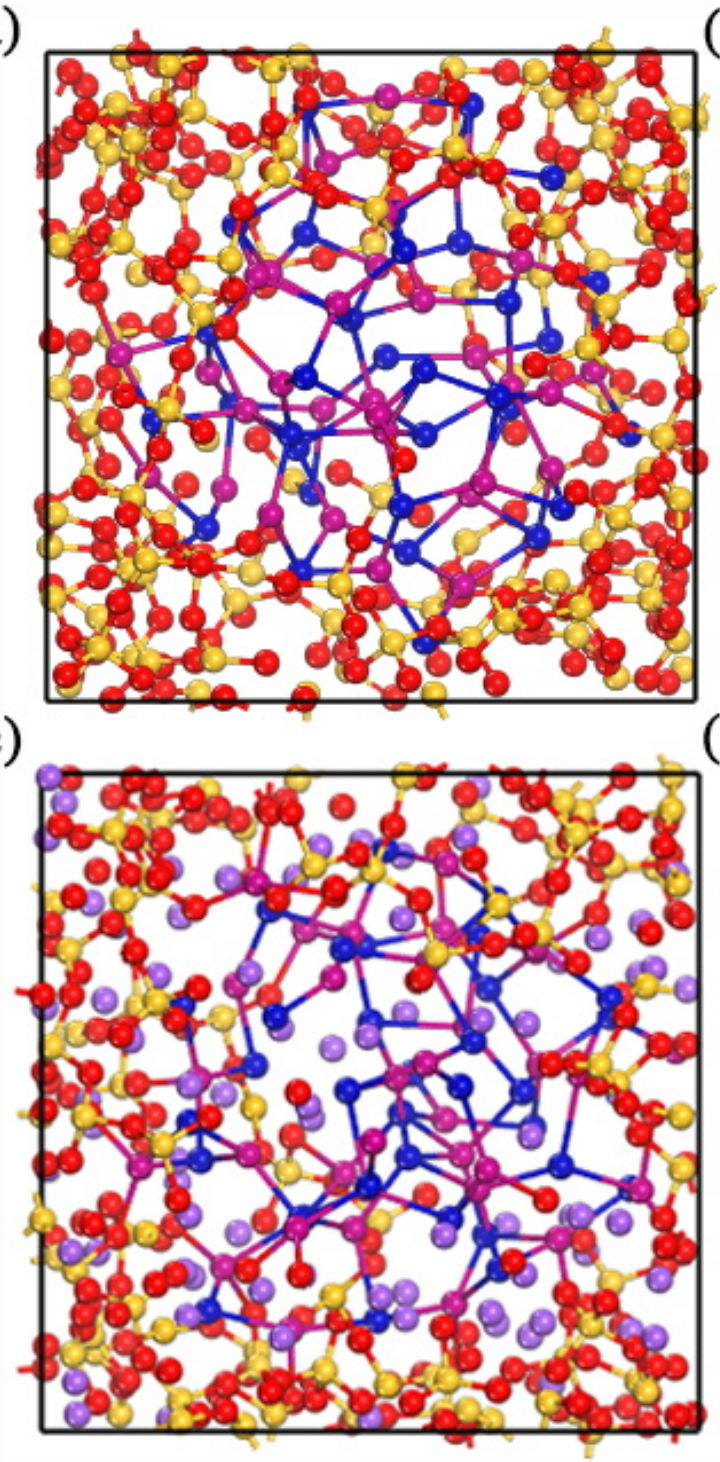

(b)

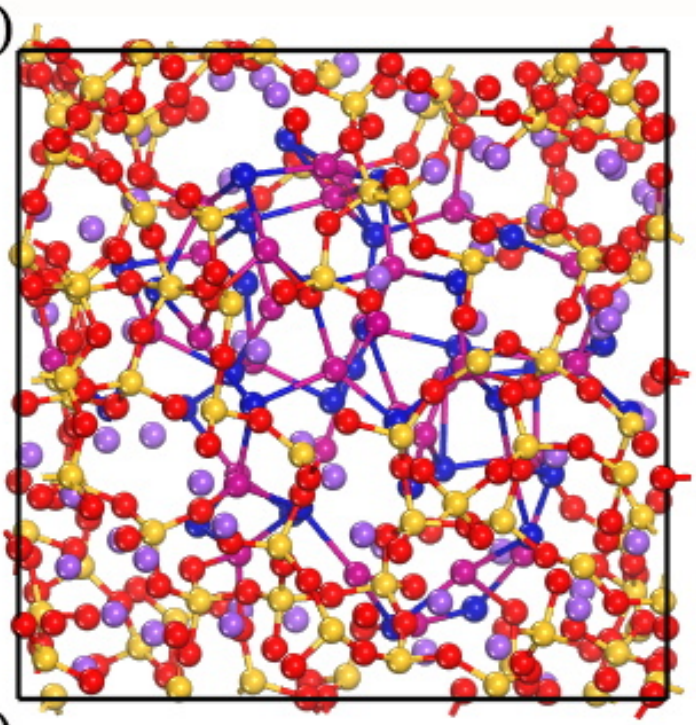

(d)

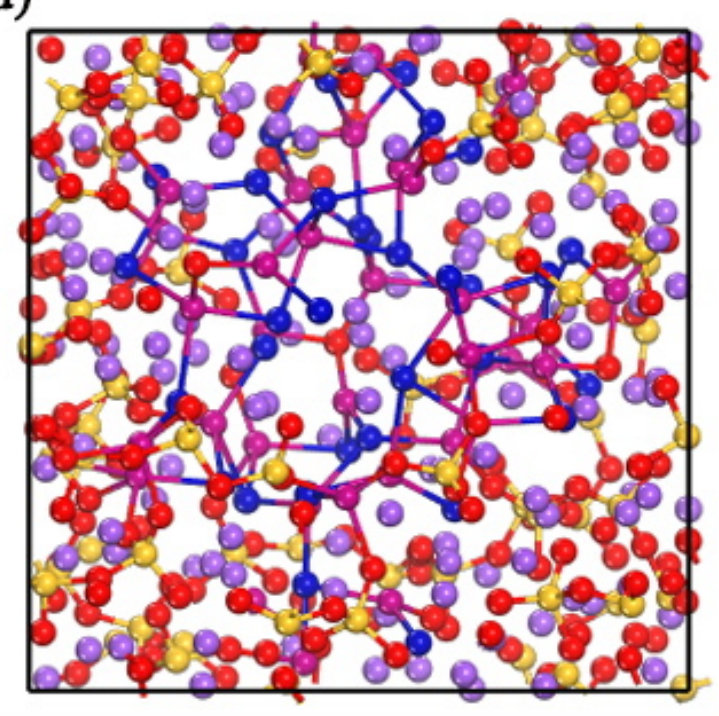

Figure 1. Final configuration from the AIMD simulations of the CdSe quantum dotdoped glasses. Glass composition: (a) $112 \mathrm{SiO}_{2}-33 \mathrm{CdSe}$, (b) $30 \mathrm{Na}_{2} \mathrm{O}-90 \mathrm{SiO}_{2}-33 \mathrm{CdSe}$, (c) $39 \mathrm{Na}_{2} \mathrm{O}-78 \mathrm{SiO}_{2}-33 \mathrm{CdSe}$, (d) $62 \mathrm{Na}_{2} \mathrm{O}-62 \mathrm{SiO}_{2}-33 \mathrm{CdSe}$. Si, yellow; O, red; Na, purple; Se, magenta; $\mathrm{Cd}$, blue.

The final structures of the CdSe quantum dot-doped glasses with different chemical composition $\left(\mathrm{Na}_{2} \mathrm{O}: \mathrm{SiO}_{2}\right.$ ratio) are depicted in Figure 1. We provide a quantitative analysis of the chemical environment of each structure in Table 2. As glass modifiers, sodium ions can break the $\mathrm{Si}-\mathrm{O}$ bond between $\mathrm{Si}$ and $\mathrm{O}$ ions, contributing to the appearance of non-bridging oxygen atoms in the inorganic network. The percentage of $\mathrm{O}$ atoms bonded with $\mathrm{Si}$ atoms decreased from $96.8 \%$ to $32.0 \%$ upon increase in the amount of $\mathrm{Na}_{2} \mathrm{O}$ in the glass. Meanwhile, the non-bridging oxygen preferred to be bonded with $\mathrm{Cd}$ atoms rather than $\mathrm{Na}$ atoms, as demonstrated by an increase of percentages of $\mathrm{O}$ atoms bonded with $\mathrm{Cd}$ atoms from $2.9 \%$ to $5.9 \%$. Se atoms also tended 
to be bonded preferentially with sodium ions as the amount of the sodium oxygen grows larger in the glass matrices, with $37.1 \%$ of Se atoms bonded with $\mathrm{Na}$ atoms when $x=$ 0.5. In some configurations, up to $33 \mathrm{Se}$ atoms were found to be bonded with sodium ions, an increased breakage of the bond between $\mathrm{Cd}$ atoms and $\mathrm{Se}$ atoms when adding sodium oxygen into the glass matrices was observed.

These results showed that, near the quantum dot/glass interface, the CdSe QD is quite sensitive to the composition of the glass matrix. This has been experimentally suspected based on the Raman spectroscopic data showing that the Raman shift of CdSe QDs with identical size encompassed in different host matrices were different. In previous work, a lattice contraction effect, depending on the thermodynamic conditions decided by the interactions between QDs and glass matrix, was proposed to explain this observation - but without further direct evidence. ${ }^{21}$ In the colloidal method, the CdSe QD core was observed to maintain its bulk structure when capped by the organic ligands. ${ }^{7,22-23}$ However, the interfacial structure and the structure of the CdSe QD reconstructed enormously in our results.

Table 2 Average percentage of $\mathrm{O}$, Na, Si Se, and $\mathrm{Cd}$ bonded with different atoms averaged over the AIMD simulations of CdSe QD in $x \mathrm{Na}_{2} \mathrm{O}-(1-x) \mathrm{SiO}_{2}$ glass matrix.

\begin{tabular}{|c|c|c|c|c|c|}
\hline & Bonds & $x=0$ & $x=0.25$ & $x=0.33$ & $x=0.5$ \\
\hline \multirow{3}{*}{$\mathbf{O}$} & O-Si & 96.79 & 59.44 & 50.29 & 31.97 \\
\hline & O-Na & & 36.82 & 44.54 & 62.16 \\
\hline & O-Cd & 2.91 & 3.72 & 5.17 & 5.87 \\
\hline $\mathbf{S i}$ & Si-O & 97.99 & 96.25 & 94.54 & 83.07 \\
\hline \multirow{2}{*}{$\mathrm{Na}$} & Na-O & & 87.45 & 86.21 & 83.57 \\
\hline & Na-Se & & 7.4 & 8.05 & 7.73 \\
\hline \multirow{2}{*}{ Se } & Se-Na & & 17.06 & 23.72 & 37.09 \\
\hline & Se-Cd & 89.61 & 81.9 & 76.26 & 62.88 \\
\hline \multirow{2}{*}{ Cd } & Cd-O & 12.88 & 19.87 & 27.7 & 35.95 \\
\hline & Cd-Se & 87.1 & 79.89 & 71.72 & 59.73 \\
\hline
\end{tabular}

First of all, we want to outline some definitions here to better illustrate the complexity of the hybrid systems considered in this work. We divide each configuration of $\mathrm{CdSe}$ quantum dot-doped glasses into three regions of space: (a) the hybrid QD; (b) the hybrid glasses, in the interfacial region; and (c) the pristine glass, at distance such that its structure is not affected by the QD (Figure 2). When the pristine CdSe QD is introduced into the glass matrix, an important structural reconstruction is observed, and we note the resulting structure as a "hybrid QD" (including Cd and Se atoms) to highlight that its structure is very different from the pristine QD. At the same time, the structural reconstruction also happens in the glass matrix due to interactions with QD, forming a "hybrid glass" (including $\mathrm{O}, \mathrm{Na}$ and $\mathrm{Si}$ atoms). The hybrid QD and hybrid glass are connected by forming interfacial bonds such as $\mathrm{Na}-\mathrm{Se}$ and $\mathrm{Cd}-\mathrm{O}$ linkages. However, the density of the QDs in the glass matrix is small, thus the incorporation of CdSe QD can only influence the glass structure at short range, without further impact on the glass 
structure in the long range. Therefore, the structure of the pristine glass which is far from the CdSe QD remains unchanged. These micro regions are separated by dense and amorphous pristine glass.

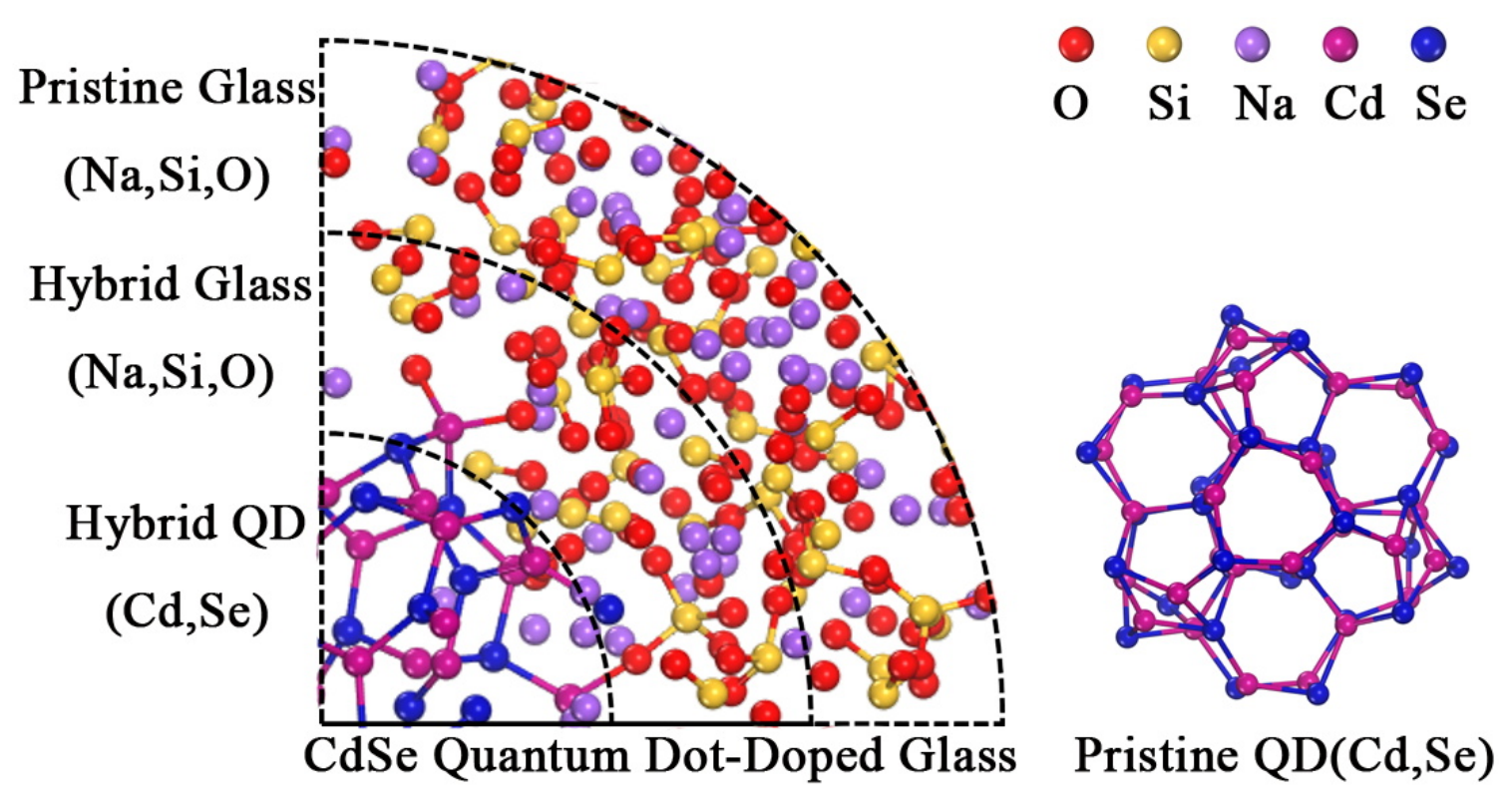

Figure 2 Illustration of structural composition of CdSe quantum dot-doped glasses.

Within our chosen methodology, the HOMO-LUMO gap for the pristine QD is found to be $2.08 \mathrm{eV}$, which is smaller than the experimental value $(\sim 3 \mathrm{eV}) .{ }^{24}$ The goal of our present work is not to reproduce the exact value of the experimentally observed absorption peak, but rather to predict the impact of the glass matrices on a relative energy scale, which can be corrected "a posteriori" by a constant energy shift to a first approximation. In order to perform significant statistical sampling of different configurations obtained from AIMD, we are also constrained by computational cost. In addition, 10 configurations were selected from the AIMD production run of pristine glasses, and the average HOMO-LUMO gaps are calculated at $7.19 \mathrm{eV}, 4.44 \mathrm{eV}, 4.33$ $\mathrm{eV}$ and $4.41 \mathrm{eV}$ when $x$ is $0,0.25,0.33$, and 0.5 , respectively. The reported experimental value for amorphous $\mathrm{SiO}_{2}$ lies in the range of 8.7 to $9.4 \mathrm{eV} .{ }^{25-26}$ The calculated HOMOLUMO gap of $\mathrm{SiO}_{2}$ glass in our work was comparable to other researchers' work, which were $5.3 \mathrm{eV}^{19}, 5.35 \mathrm{eV}^{27}, 5.657 \mathrm{eV}^{28}$, and $8.47 \mathrm{eV}^{29}$, depending on the computational methodology. When $\mathrm{Na}_{2} \mathrm{O}$ is introduced into pure $\mathrm{SiO}_{2}$, first the band gap decreases sharply but the decreasing rate slows down after $x=0.2 .^{28}$ The gap of pristine glass with $x=0.5$ was higher than that with $x=0.33$, which may be due to strained regions after structure relaxation in $x=0.5$ model $^{29}$

50 configurations were chosen from the AIMD production runs of the CdSe quantum dot-doped glasses in order to conduct further static DFT calculations. The HOMOLUMO gap distribution of each composition was shown in Figure 3. The average values of the gap were $1.95 \mathrm{eV}, 2.11 \mathrm{eV}, 1.71 \mathrm{eV}$ and $1.84 \mathrm{eV}$, when $x$ changed from 0 (Figure 3(a)), 0.25 (Figure 3(b)), 0.33 (Figure 3(c)) and 0.5 (Figure 3(d)), respectively. The relationship between the HOMO-LUMO gap and the ratio of the 
$\mathrm{Na}_{2} \mathrm{O}$ is nonlinear, and different from the relationship between the HOMO-LUMO gap of the pristine glass and sodium oxygen contents.
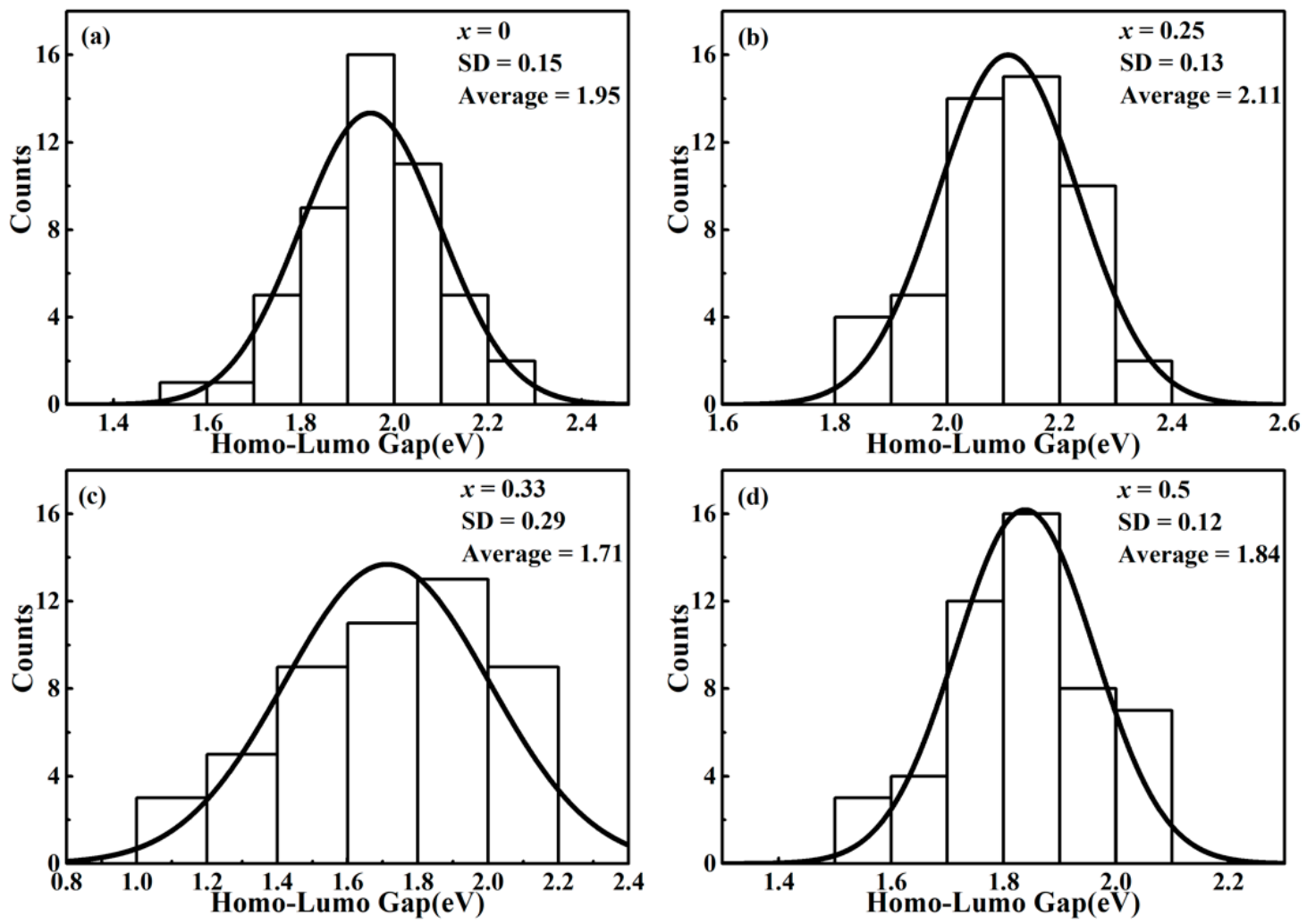

Figure 3 Histograms of HOMO-LUMO gap for CdSe quantum dot-doped sodium silicate glasses. The composition of the $x \mathrm{Na}_{2} \mathrm{O}-(1-x) \mathrm{SiO}_{2}$ glass matrices: (a) $x=0$; (b) $x=0.25$; (c) $x=0.33$; (d) $x=0.5$. A Gaussian function was used to fit the data and SD represent the standard deviation.

Experimentally fabricated glasses are inhomogeneous in microscopic regions due to structure fluctuations in such disordered materials, in addition to the local fluctuations in the composition and thermal conditions. With our statistical sampling of configurations from the molecular dynamics trajectory, we can represent this diversity in structure (and, as a consequence, physical and chemical properties) despite having a relatively small simulation box: each configuration in our calculation can represent a small part of the real glass sample, representing different possible interfacial environments of $\mathrm{CdSe}$ quantum dots in the glass matrices. The optical properties of the glass sample are therefore a statistical representation of these micro regions. Even though the composition for each configuration of the same system identical, we find that the HOMO-LUMO gap still displays important fluctuations, with standard deviations of $0.15,0.13,0.29$ and $0.12 \mathrm{eV}$, respectively. These results demonstrate that the interfacial environment of each QD in glass matrices is rather complicated in terms of the electronic structures.

It should be noted that the standard deviation of $x=0.33$ was much higher than other 
glasses composition, with an asymmetric distribution featuring a long tail at low values. Therefore, it indicates broad distribution in absorption and photoluminescence of CdSe QD formed in the glasses, resulting in low photoluminescence quantum yield of QDs in glasses, which is unfavorable for their potential application. Thus, it gives guidance to the compositional design of glass matrix that $x=0.33$ is not a desirable ratio if we want to fabricate highly luminescent glasses.
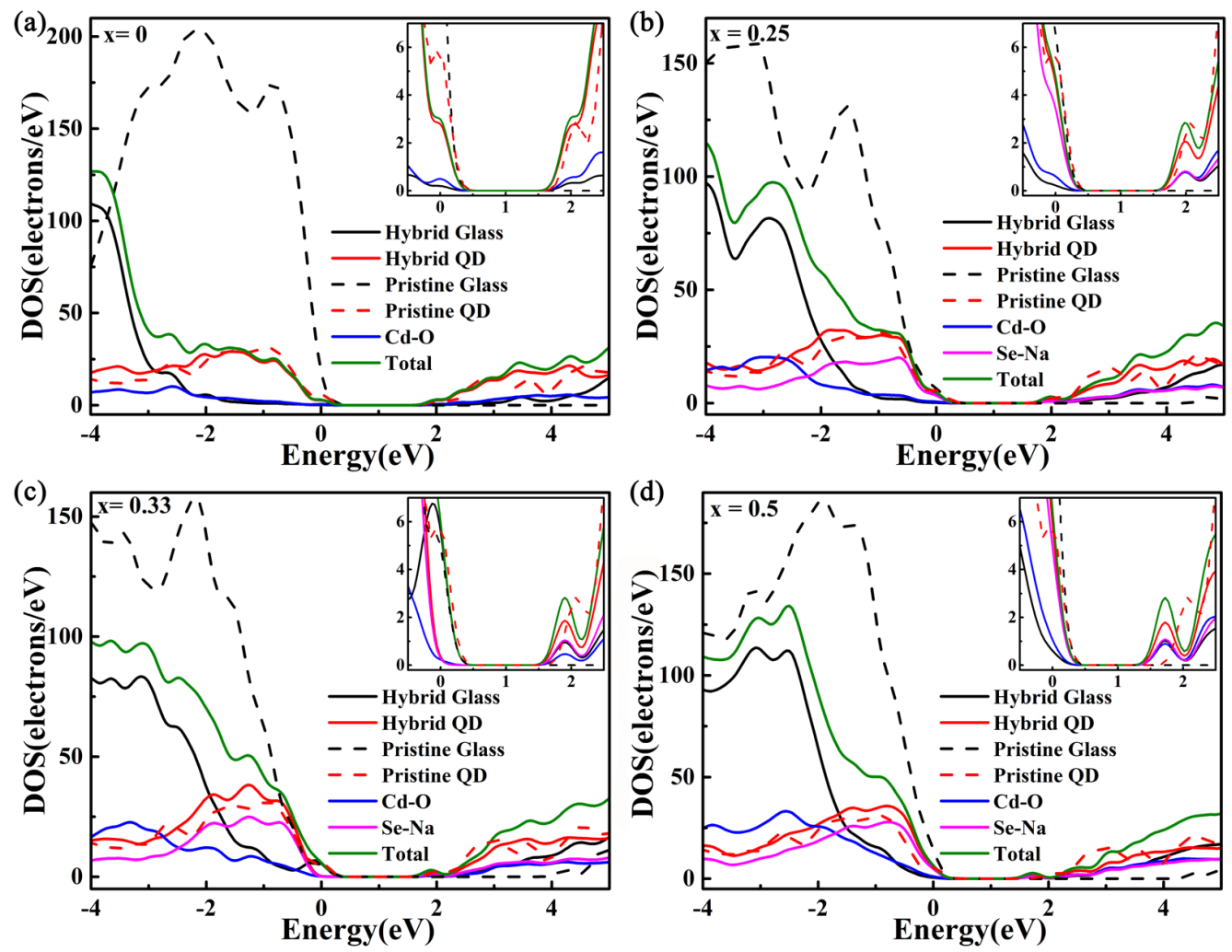

Figure 4 Density of states and projected density of states of final structure of the AIMD calculation of CdSe quantum dot-doped glasses. The hybrid glass included $\mathrm{O}$ atoms, $\mathrm{Na}$ atoms and $\mathrm{Si}$ atoms, while hybrid QD was composed of $\mathrm{Cd}$ atoms and $\mathrm{Se}$ atoms. $\mathrm{Cd}-\mathrm{O}$ (and $\mathrm{Se}-\mathrm{Na}$ ) represent the projected density of states of the $\mathrm{Cd}$ (respectively, $\mathrm{Se}$ ) atoms bonded with $\mathrm{O}(\mathrm{Na})$ atoms and $\mathrm{O}(\mathrm{Na})$ atoms bonded with $\mathrm{Cd}(\mathrm{Se})$ atoms. The HOMO and the Fermi level are set at $0 \mathrm{eV}$. Panel (a) to (d) correspond to compositions of $x=0,0.25,0.33$, and 0.5 , respectively.

In order to have a better understanding of the impact of glass matrices on the electronic structures of the CdSe QD, the density of states and projected density of states of the pristine glass, pristine $\mathrm{QD}$, and CdSe quantum dot-doped glass were calculated and are plotted on Figure 4. For the final structure of $x=0$ (Figure 4(a)), $x=0.25$ (Figure 4(b)) and $x=0.5$ (Figure 4(d)), the HOMO orbitals and LUMO orbitals are determined by the hybrid QD. Interestingly, the HOMO orbitals in $x=0.33$ (Figure 4(c)) appear different compared to other composition, because it is determined by hybrid glass instead of the hybrid QD, while, the LUMO orbitals are still decided by hybrid QD. These results demonstrate two different luminescence mechanism of CdSe quantum 
dot-doped glasses, which we schematize on Figure 5. For what we will call type I (Figure 5(a)), when the electrons are excited from the hybrid glass, they will leave holes on the valence band. These photogenerated electrons will relax to the LUMO orbitals formed by the hybrid QD. The holes and electrons are in different spatial locations, resulting in indirect recombination (red straight dash line) and enhanced nonradiative recombination (red curve dash line). For what we will call Type II (Figure 5(b)), both electrons and holes are generated in hybrid QD upon light excitation, leading to the direct recombination(red straight line) and subsequent intrinsic emission from hybrid QD.

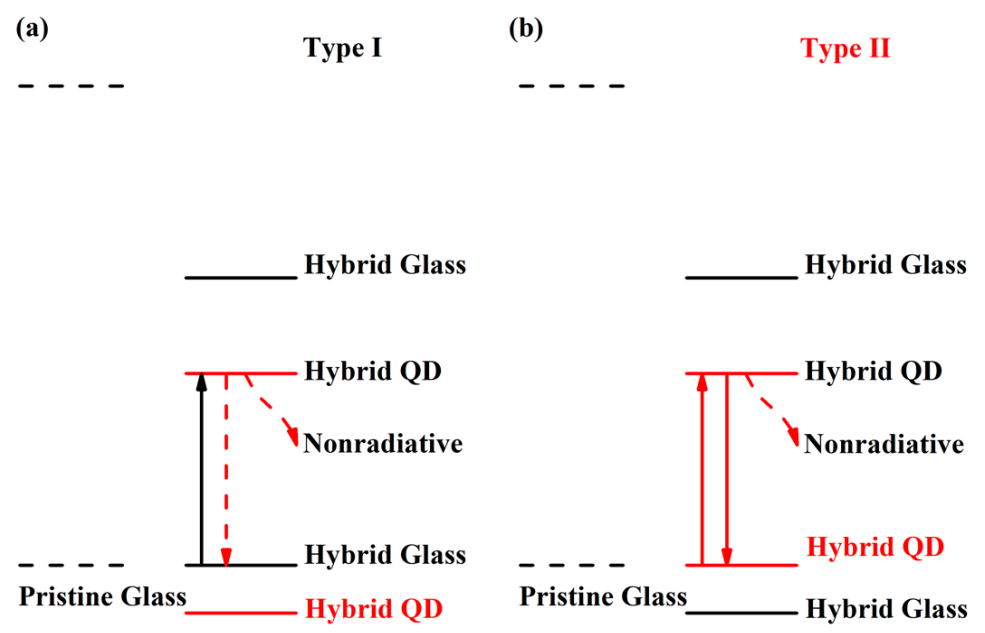

Figure 5 Energy diagrams demonstrating two different luminescence mechanism of CdSe quantum dot-doped glasses.

In addition, we also looked deeper in the impact of interfacial bonds. As we can clearly see from Figure 4, the projected density of states of Se-Na (magenta solid line ) and $\mathrm{Cd}-\mathrm{O}$ (blue line) contribute to the peak at the bottom of conduction band. However, the peak formed by the hybrid QD (red solid line), Se-Na, and $\mathrm{Cd}-\mathrm{O}$ are at the same position. Therefore, we can conclude that both $\mathrm{Se}-\mathrm{Na}$ and $\mathrm{Cd}-\mathrm{O}$ linkages contribute to the frontier orbitals but do not shift their energy level.

We further analyzed the density of states of 50 individual configurations of each composition. Based on the DOS, each configuration was assigned to one of the types of luminescence mechanism described above (Figure 6). Importantly, the luminescence mechanisms of CdSe quantum dot-doped glasses were found to be strongly dependent on the composition of the glass matrices. When $x=0,0.25$, or 0.5 , the luminescence was mainly determined by the direct transition between HOMO and LUMO of hybrid QD (type II mechanism). On the other hand, when $x=0.33$, the luminescence was mainly determined by the indirect transition between the LUMO of hybrid QD and HOMO of hybrid glass (type I mechanism), and only 7 out of 50 configurations were classified to type II. Thus, we see again that the composition of the glass with $x=0.33$ is less favorable for practical applications. For one thing, the standard deviation of $x=$ 0.33 (Figure 3) is much higher than other compositions, contributing to the broad 
distribution of photoluminescence. In addition to that, the electrons and holes are generated in different spatial regions, tending to induce indirect recombination. Both effects are likely to reduce the quantum efficiency.
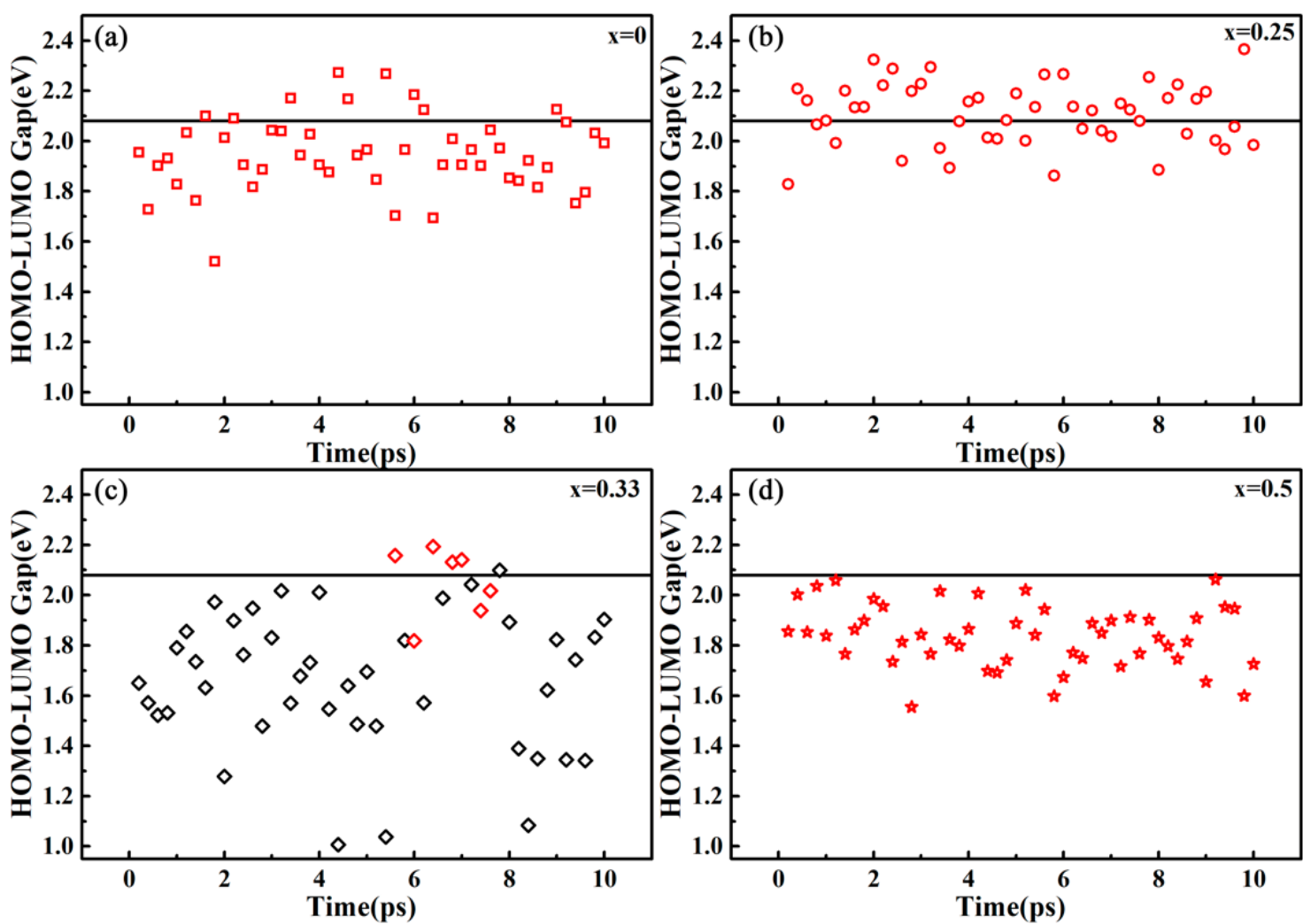

Figure 6 HOMO-LUMO gap of the 50 configurations of the AIMD production run of CdSe quantum dot-doped glass. The solid black line represent the HOMO-LUMO gap of the pristine QD. The black points represent the luminescence mechanism of this configuration belonging to type I and red points represent type II.

Traditionally, the luminescence of the CdSe quantum dot-doped glass can be divided into two competitive parts, which are defect emission and intrinsic emission. ${ }^{5,} 30$ The defect emission is attributed to trap states formed by surface defects such as dangling bond, vacancies and so on. Meanwhile, the intrinsic emission of CdSe QD is related to the direct recombination of the excitons formed within the QD.

However, according to the atomic structure and the HOMO-LUMO gap as well as the density of states of each configuration, we can establish a new luminescence mechanism quite different from the models proposed in previously published work. We hypothesize that the luminescence of CdSe quantum dots embedded in silicate glass matrices observed in experiments, was not defect emission induced by the surface defects, nor intrinsic emission from pristine QD as is commonly proposed, but the intrinsic emission of the hybrid QD and hybrid glasses.

It is a highly hybrid system, in which the surface defects and core cannot be separately 
discussed because their coupling is too important due to strong reorganization at the interface. Firstly, we analyzed the structural properties of the 200 configurations at all compositions, and large-scale structure reconstruction can be observed in each configuration: it is hard for the core to maintain its bulk crystalline structure. $\mathrm{Na}$ atoms were found to be bonded with the Se atoms even in the core of the QD. Therefore, there is little possibility for the CdSe QD keep a "pristine" state. Secondly, only a small ratio of configurations were found to have the same value of HOMO-LUMO gap as the pristine QD (Figure 6) with a broad distribution of values instead, further proving the little possibility of the existence of pristine QD in glass matrices. Thirdly, no midgap state was found to be formed inside the HOMO-LUMO gap, as observed in some colloidal quantum dots ${ }^{7,9-10,31-32}$ where the orbital of pristine QD still exists. Therefore, pristine QD is less likely to exist in the sodium silicate glass. The emission of the CdSe quantum dot-doped glasses originated from the exciton recombination of the hybrid system formed by the hybrid QD and hybrid glass, and cannot be described by simplistic models.

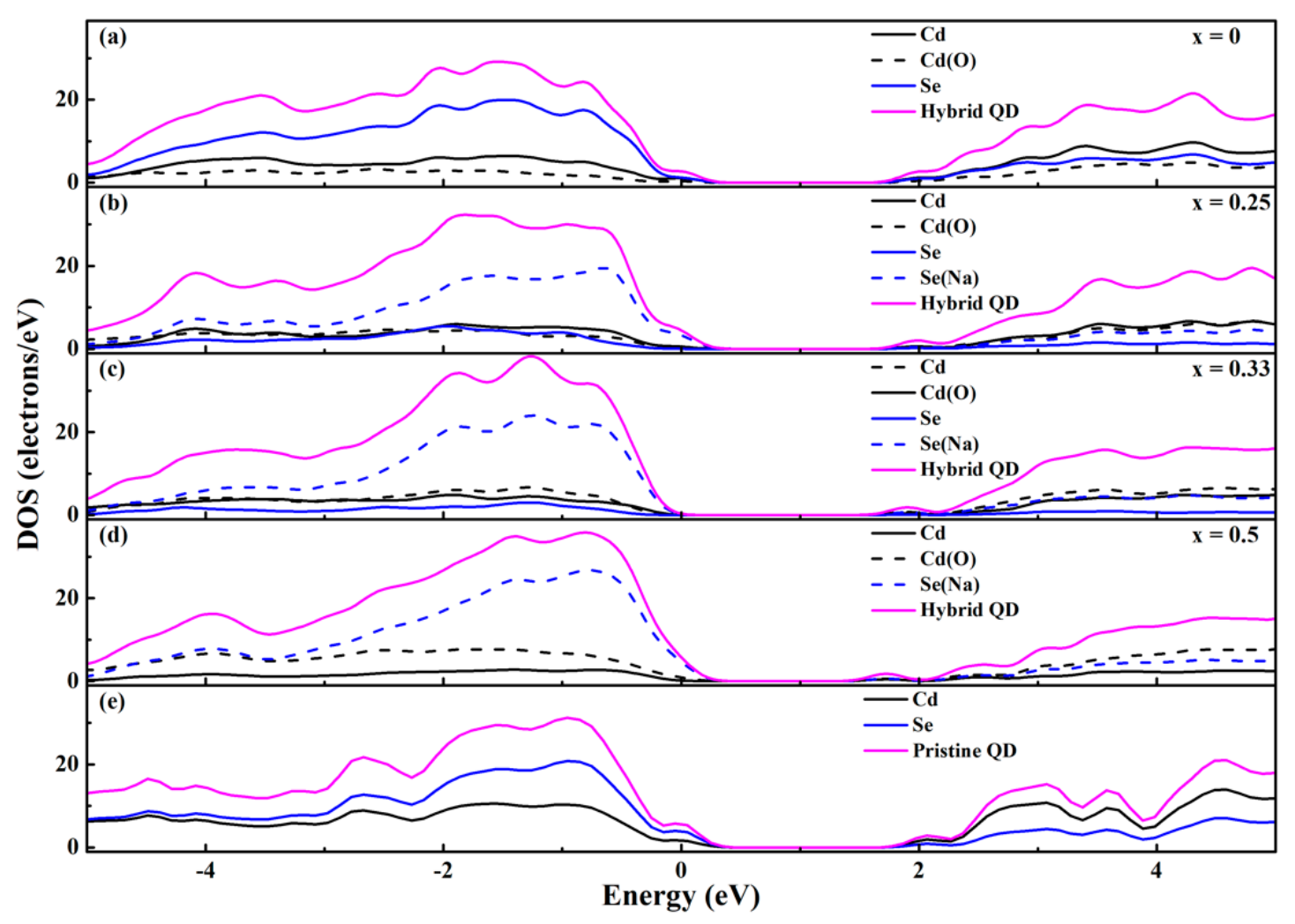

Figure 7 The density of states of the final structure of AIMD simulation of CdSe quantum dot-doped glasses and pristine $\mathrm{QD} . \mathrm{Cd}(\mathrm{O})$ represents the $\mathrm{Cd}$ atoms bonded with $\mathrm{O}$ atoms and $\mathrm{Se}(\mathrm{Na})$ represents the $\mathrm{Se}$ atoms bonded with $\mathrm{Na}$ atoms. Cd represents the $\mathrm{Cd}$ atoms not bonded with $\mathrm{O}$ atoms. Se represent the Se atoms not bonded with $\mathrm{Na}$ atoms.

We further investigate the impact of the glass matrix (and its composition ) on electronic structure of the CdSe quantum dot (Figure 7) by comparing the density of states of 
hybrid and pristine QDs. The top of the valence band of the pristine QD is determined by Se atoms and the bottom of conduction band by $\mathrm{Cd}$ atoms (Figure 7(e)). Upon introduction of the quantum dot into the glass matrix, the top of the valence band remains determined by $\mathrm{Se}$, but the Se atoms bonded with $\mathrm{Na}$ atoms are found to be the main contributors to the frontier orbitals. It should be noted that all Se atoms were bonded with $\mathrm{Na}$ atoms in the final structure of $x=0.5$ (Figure 7(d)). The bottom of the conduction band is determined by $\mathrm{Cd}$ atoms and the $\mathrm{Cd}$ atoms not bonded with $\mathrm{O}$ atoms contributed mostly to the LUMO.
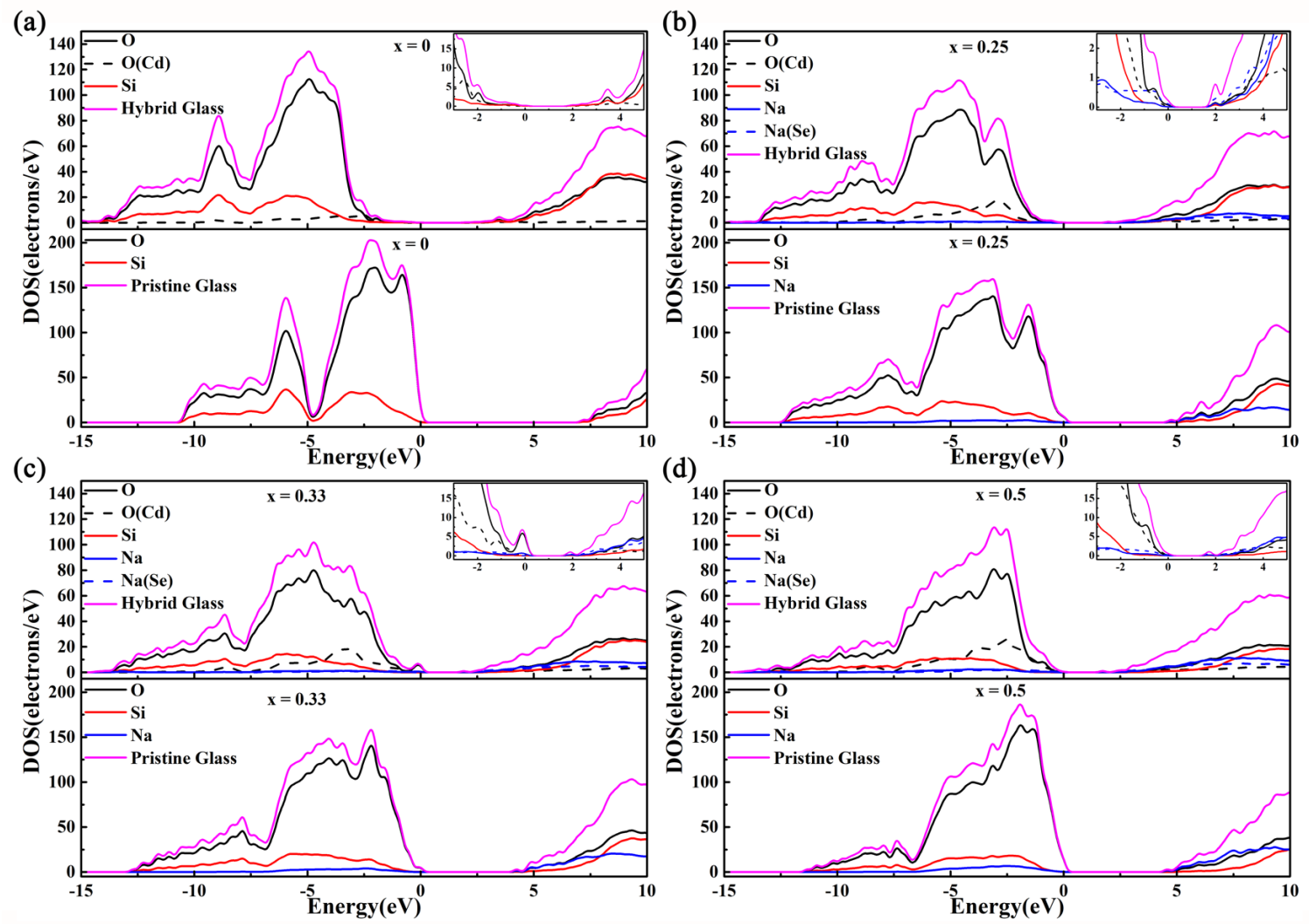

Figure 8 The density of states of the final structure of the AIMD simulation of CdSe quantum dot-doped glasses and pristine glasses. $\mathrm{O}(\mathrm{Cd})$ represents the $\mathrm{O}$ atom bonded with $\mathrm{Cd}$ atom and $\mathrm{Na}(\mathrm{Se})$ represent the $\mathrm{Na}$ atom bonded with $\mathrm{Se}$ atom.

Finally, we investigated the effect of the presence of the CdSe QDs on the electronic structure of the glasses. There are two typical segments on the upper valence band of pristine glasses as well as the hybrid glasses (Figure 8). They mainly consist in oxygen $2 p$ lone pair orbitals of for the highest orbitals and in bonding states between silicon $s p^{3}$ hybrids and oxygen $2 p$ orbitals from $-10 \mathrm{eV}$ to $-5 \mathrm{eV} \cdot{ }^{33-34}$ It is apparent that the introduction of CdSe QD sharply decreases the gap from that of the pristine glass, with the bottom of the conduction band shifting to the lower energy part. Besides, an additional peak appears at the shoulder of the upper segments formed by the oxygen lone pair orbitals in CdSe quantum dot-doped glasses, which can be attributed to nonbonding oxygen. 

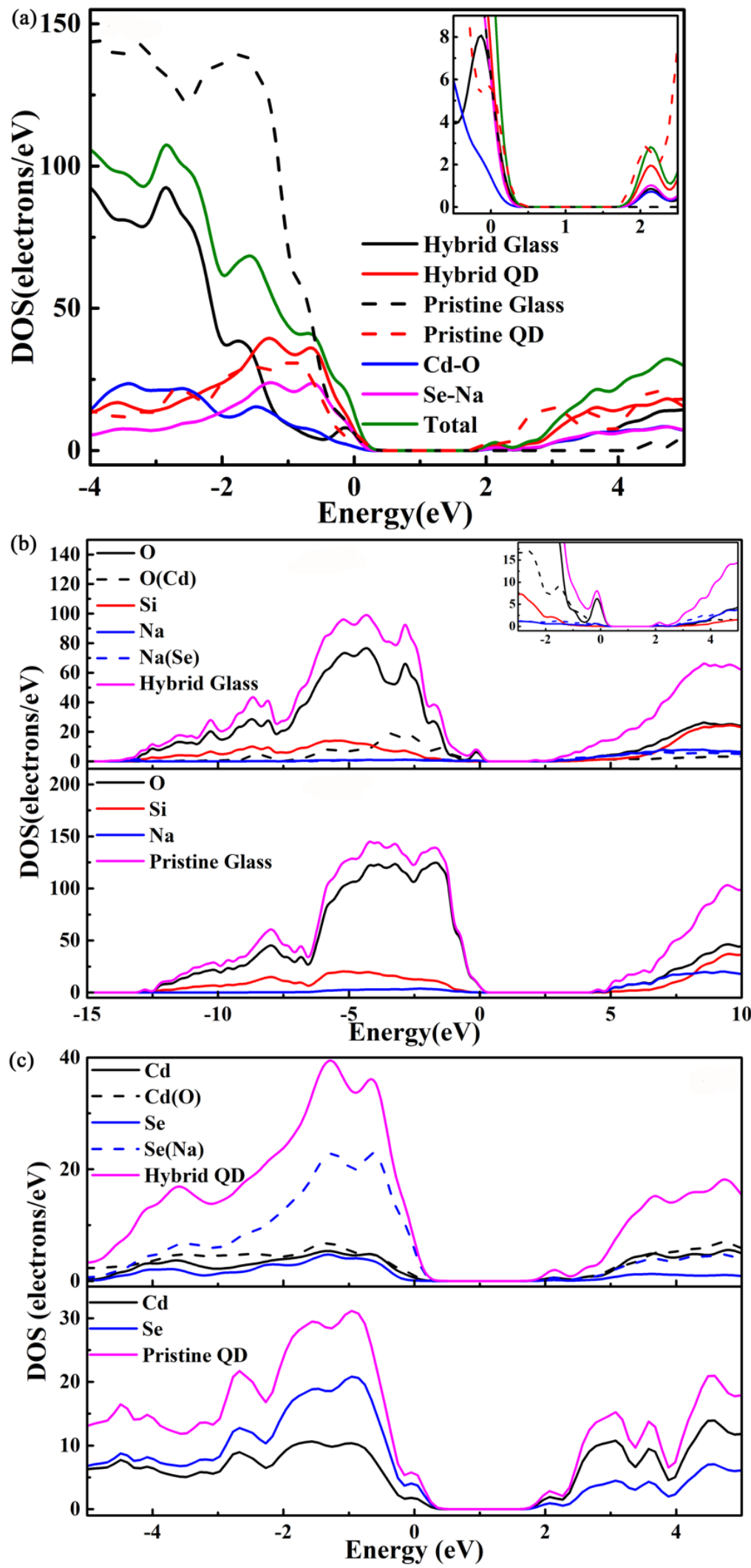

Figure 9 The configuration was obtained from the AIMD production run with $x=0.33$ at 7 ps. (a) The density of states of hybrid glass, hybrid QD, pristine QD, pristine glass $(x=0.33), \mathrm{Cd}-\mathrm{O}$ bonds and Se-Na bonds. (b) The density of states of the hybrid glasses and pristine glasses $(x=0.33)$. (c) The density of states of the hybrid QD and the pristine 
QD.

Although we identified 7 configurations (red points in Figure 6(c)), out of 50, for which the luminescence mechanism belongs to type II when $x=0.33$, the impact of the CdSe QD on the pristine glass and the effect of the pristine glass on CdSe is qualitatively the same as other configurations. For example, the configuration obtained from the AIMD production run at 7 ps with $x=0.33$, it is apparent that the top of the valence band is determined by the hybrid QD, so the luminescence mechanism of this configuration is assigned to type II (Figure 9(a)), different from the majority of the configurations of $x$ $=0.33$. However, the impact of glass or CdSe QD demonstrates similar tendency. A much smaller HOMO-LUMO gap of hybrid glasses compared with pristine glasses (Figure 9(b)) can be observed, indicating the presence of CdSe QD can greatly decrease the bandgap of pristine QD. The hybrid orbitals formed by Se atoms and Na atoms played a dominate role in the HOMO orbitals. Meanwhile, similarly, the HOMO orbital is found to be decided by Se atoms which are bonded with $\mathrm{Na}$ atoms in hybrid QD (Figure 9(c)).

\section{Conclusions and Perspectives}

To the best of our knowledge, we present here the first realistic electronic structure calculations of CdSe quantum dot-doped glasses. By varying the amount of the sodium oxygen in $x \mathrm{Na}_{2} \mathrm{O}-(1-x) \mathrm{SiO}_{2}$ glass matrices, the compositional dependency of structural reconstruction and luminescence mechanisms was illustrated, which is of prominent importance for exploring these materials' optical properties.

The additional introduction of $\mathrm{Na}_{2} \mathrm{O}$ in the glass matrix is shown to promote the formation of $\mathrm{Cd}-\mathrm{O}$ bonds and $\mathrm{Se}-\mathrm{Na}$ bonds, breaking some $\mathrm{Cd}-\mathrm{Se}$ bonds. Meanwhile, the luminescence mechanisms show an important dependency on the composition of the glass matrix surrounding the quantum dot. When $x=0,0.25,0.5$, the HOMO is decided by the hybrid QD, while the HOMO is determined by the hybrid glasses in most configurations for $x=0.33$.

The presence of the CdSe quantum dot in the glass sharply decreases the bandgap of the interfacial glass (near the CdSe QD) compared with pristine glasses. The interfacial bonds contributes greatly to the frontier orbitals, and in particularly we do not observe the formation of any midgap state within the HOMO-LUMO gap. Besides, the HOMO-LUMO gap show a broader distribution at $x=0.33$, compared with other compositions. The spatial separation of the holes and electrons in most configurations of $x=0.33$ and its wide HOMO-LUMO distribution predict a low quantum efficiency of this composition.

In particular, given the atomic structure, HOMO-LUMO gap distribution and density of states of the CdSe quantum dot-doped glasses, it is apparent that there is little possibility of the existence of the pristine QD, demonstrating no intrinsic emission from the pristine QD. Thus, a totally new energy diagram model is proposed, very different 
from that known for colloidal QDs, and also from the model generally proposed in the literature for $\mathrm{CdSe}$ quantum dot-doped glasses (based on indirect experimental evidence). The CdSe QD is surrounded by the glass matrix, where the structural reconstruction happens, forming the structure of hybrid QD and hybrid glass. Due to the low concentration of the QDs in glass matrices, these hybrid system only exist in nano-scale regions, surrounded by dense amorphous pristine glass, acting as a shell towards the hybrid systems composed of hybrid QD and hybrid glasses. Therefore, the emission of the CdSe quantum dot-doped glasses originate from the intrinsic emission of the hybrid systems, rather than the intrinsic and defect emission from the pristine QD and its trap states in the classical model.

Our results help explain why the quantum efficiency of CdSe quantum dot-doped glass is much lower than their colloidal counterparts. On the one hand, there is no intrinsic emission from the pristine QD due to the strong impact of the glass matrices on CdSe QD. On the other hand, from the experimental part, the glass is inhomogeneous in micro regions due to the fluctuation of the thermal condition and composition. Even with the same composition of these hybrid systems, the electronic structures of each configuration was variable, exhibiting different optical properties, resulting in the broad emission.

Our results demonstrate the crucial importance of the composition of glass matrix, giving a straightforward illustration of the electronic structure of CdSe quantum dotdoped glasses, enhancing the mature understanding of its luminescence mechanism from a different perspective. Especially, these luminescence mechanism can be altered by varying the sodium content in glass matrices. We expect, from the results obtained in our work, the composition of $x=0.33$ will exhibit poor quantum efficiency. Meanwhile, the composition of $x=0.5$ is not stable due to the high sodium contents in glass matrices and the melting point of $x=0$ is much too high. Therefore, the composition of $x=0.25$ will be an ideal composition for the glass matrices, with holes and electrons confined in the hybrid QD, higher average HOMO-LUMO gap compared with pristine QD and narrow HOMO-LUMO gap distribution.

\section{Corresponding authors}

* hite@,whut.edu.cn; fx.coudert@chimieparistech.psl.eu

\section{Acknowledgements}

This work was financially supported by the Natural Science Foundation of Hubei Province (2018CFA005) and the China Scholarship Council (201806950059). We acknowledge access to high-performance computing platforms provided by GENCI grant A0090807069.

\section{References}

1. Fan, F.; Voznyy, O.; Sabatini, R. P.; Bicanic, K. T.; Adachi, M. M.; McBride, J. R.; Reid, K. R.; Park, Y. S.; Li, X.; Jain, A.; Quintero-Bermudez, R.; Saravanapavanantham, 
M.; Liu, M.; Korkusinski, M.; Hawrylak, P.; Klimov, V. I.; Rosenthal, S. J.; Hoogland, S.; Sargent, E. H., Continuous-Wave Lasing in Colloidal Quantum Dot Solids Enabled by Facet-Selective Epitaxy. Nature 2017, 544 (7648), 75-79.

2. Yang, Y.; Zheng, Y.; Cao, W.; Titov, A.; Hyvonen, J.; Manders, J. R.; Xue, J.; Holloway, P. H.; Qian, L., High-Efficiency Light-Emitting Devices Based on Quantum Dots With Tailored Nanostructures. Nat. Photonics 2015, 9 (4), 259-266.

3. Wichner, S. M.; Mann, V. R.; Powers, A. S.; Segal, M. A.; Mir, M.; Bandaria, J. N.; DeWitt, M. A.; Darzacq, X.; Yildiz, A.; Cohen, B. E., Covalent Protein Labeling and Improved Single-Molecule Optical Properties of Aqueous CdSe/CdS Quantum Dots. ACS Nano 2017, 11 (7), 6773-6781.

4. Han, K.; Im, W. B.; Heo, J.; Chung, W. J., A Complete Inorganic Colour Converter Based on Quantum-Dot-Embedded Silicate Glasses for White Light-Emitting-Diodes. Chem Commun (Camb) 2016, 52 (17), 3564-7.

5. Han, K.; Yoon, S.; Chung, W. J., CdS and CdSe Quantum Dot-Embedded Silicate Glasses for LED Color Converter. Int. J. Appl.Glass Sci. 2015, 6 (2), 103-108.

6. Abuelela, A. M.; Mohamed, T. A.; Prezhdo, O. V., DFT Simulation and Vibrational Analysis of the IR and Raman Spectra of a CdSe Quantum Dot Capped by Methylamine and Trimethylphosphine Oxide Ligands. J. Phys. Chem. C 2012, 116 (27), 14674-14681. 7. Tamukong, P. K.; Peiris, W. D.; Kilina, S., Computational Insights into CdSe Quantum Dots' Interactions with Acetate Ligands. Phys. Chem. Chem. Phys. 2016, 18 (30), 20499-510.

8. Voznyy, O.; Sargent, E. H., Atomistic Model of Fluorescence Intermittency of Colloidal Quantum Dots. Phys. Rev. Lett. 2014, 112 (15), 157401.

9. Kuznetsov, A. E.; Beratan, D. N., Structural and Electronic Properties of Bare and Capped $\mathrm{Cd}_{33} \mathrm{Se}_{33}$ and $\mathrm{Cd}_{33} \mathrm{Te}_{33}$ Quantum Dots. J. Phys. Chem. C 2014, 118 (13), 70947109.

10. Puzder, A.; Williamson, A. J.; Gygi, F.; Galli, G., Self-Healing of CdSe Nanocrystals: First-Principles Calculations. Phys. Rev. Lett. 2004, 92 (21), 217401.

11. Lipatova, Z. O.; Kolobkova, E. V.; Babkina, A. N., Luminescent Properties of Cadmium Selenide Quantum Dots in Fluorophosphate Glasses. Opt. Spectrosc. 2016, 121 (5), 713-721.

12. Li, W.; Li, N.; Liu, C.; Greaves, G. N.; Ong, W. J.; Zhao, X., Understanding the Atomic and Electronic Structures Origin of Defect Luminescence of CdSe Quantum Dots in Glass Matrix. J. Am. Ceram. Soc. 2019, 102 (9), 5375-5385.

13. Li, W.; Zhao, X.; Liu, C.; Coudert, F. X., Ab Initio Molecular Dynamics of CdSe Quantum-Dot-Doped Glasses. J. Am. Chem. Soc. 2020, 142 (8), 3905-3912.

14. Choi, J.; Park, B. J.; Choi, Y. G.; Heo, J.; Chung, W. J., Compositional Dependence of $\mathrm{Se}_{2}^{-}$Color Center Formation in Silicate Glasses. J. Non-Cryst. Solids 2013, 377, 70-73.

15. Adamo, C.; Barone, V., Toward Reliable Density Functional Methods without Adjustable Parameters: The PBE0 Model. J. Chem. Phys. 1999, 110 (13), 6158-6170.

16. Wang, X.; Zeng, Q.; Shi, J.; Jiang, G.; Yang, M.; Liu, X.; Enright, G.; Yu, K., The Structure and Optical Absorption of Single Source Precursors for II-VI Quantum Dots. Chem. Phys. Lett. 2013, 568-569, 125-129. 
17. Nadler, R.; Sanz, J. F., Simulating the Optical Properties of CdSe Clusters Using the RT-TDDFT Approach. Theor. Chem. Acc. 2013, 132, 203-211.

18. Nguyen, K. A.; Pachter, R.; Day, P. N., Computational Prediction of Structures and Optical Excitations for Nanoscale Ultrasmall $\mathrm{ZnS}$ and CdSe Clusters. J. Chem. Theory Comput. 2013, 9 (8), 3581-96.

19. Konstantinou, K.; Duffy, D. M.; Shluger, A. L., Structure and Luminescence of Intrinsic Localized States in Sodium Silicate Glasses. Phys. Rev. B 2016, 94 (17).

20. Guidon, M.; Hutter, J.; VandeVondele, J., Auxiliary Density Matrix Methods for Hartree-Fock Exchange Calculations. J. Chem. Theory Comput. 2010, 6 (8), 2348-64.

21. Hwang, Y.-N.; Shin, S.; Park, H. L.; Park, S.-H.; Kim, U., Effect of Lattice Contraction on the Raman Shifts of CdSe Quantum Dots in Glass Matrices. Phys. Rev. $B$ 1996, 54 (21), 15120-15124.

22. Troparevsky, M. C.; Kronik, L.; Chelikowsky, J. R., Optical Properties of CdSe Quantum Dots. J. Chem. Phys. 2003, 119 (4), 2284-2287.

23. Del Ben, M.; Havenith, R. W. A.; Broer, R.; Stener, M., Density Functional Study on the Morphology and Photoabsorption of CdSe Nanoclusters. J. Phys. Chem. C 2011, 115 (34), 16782-16796.

24. Kasuya, A.; Sivamohan, R.; Barnakov, Y. A.; Dmitruk, I. M.; Nirasawa, T.; Romanyuk, V. R.; Kumar, V.; Mamykin, S. V.; Tohji, K.; Jeyadevan, B.; Shinoda, K.; Kudo, T.; Terasaki, O.; Liu, Z.; Belosludov, R. V.; Sundararajan, V.; Kawazoe, Y., UltraStable Nanoparticles of CdSe Revealed from Mass Spectrometry. Nat. Mater. 2004, 3 (2), 99-102.

25. D.L.Griscom, The Electronic Structure of $\mathrm{SiO}_{2}$ : A Review of Recent Spectroscopic and Theoretical Advances. J. Non-Cryst. Solids 1977, 24 (2), 155-234.

26. G.HSiegelJr., Ultraviolet Spectra of Silicate Glasses: A Review of Some Experimental Evidence. J. Non-Cryst. Solids 1974, 13 (3), 372-398.

27. Tilocca, A.; de Leeuw, N. H., Structural and Electronic Properties of Modified Sodium and Soda-Lime Silicate Glasses by Car-Parrinello Molecular Dynamics. $J$. Mater. Chem. 2006, 16 (20), 1950-1955.

28. Baral, K.; Ching, W.-Y., Electronic Structures and Physical Properties of $\mathrm{Na}_{2} \mathrm{O}$ Doped Silicate Glass. J. Appl. Phys. 2017, 121 (24).

29. Murray, R. A., Electronic Structure of Sodium Silicate Glasses. J. Non-Cryst. Solids 1987, 95, 144-159.

30. Xia, M.; Liu, C.; Zhao, Z.; Wang, J.; Lin, C.; Xu, Y.; Heo, J.; Dai, S.; Han, J.; Zhao, X., Surface Passivation of CdSe Quantum Dots in All Inorganic Amorphous Solid by Forming $\mathrm{Cd}_{1-x} \mathrm{Zn}_{x}$ Se Shell. Sci. Rep. 2017, 7, 42359.

31. Giansante, C.; Infante, I., Surface Traps in Colloidal Quantum Dots: A Combined Experimental and Theoretical Perspective. J. Phys. Chem. Lett. 2017, 8 (20), 5209-5215. 32. Ganesan, P.; Lakshmipathi, S., Impact of Heterogeneous Passivation of Trimethylphosphine Oxide and Di-Methylphosphine Oxide Surface Ligands on the Electronic Structure of $\mathrm{Cd}_{\mathrm{n}} \mathrm{Se}_{\mathrm{n}}(\mathrm{n}=6,15)$ Quantum Dots: A DFT study. Physica E 2016, 83, 284-296.

33. Ispas, S.; Benoit, M.; Jund, P.; Jullien, R., Structural and Electronic Properties of the Sodium Tetrasilicate Glass $\mathrm{Na}_{2} \mathrm{Si}_{4} \mathrm{O}_{9}$ from Classical and Ab Initio Molecular 
Dynamics Simulations. Phys. Rev. B 2001, 64 (21).

34. Du, J.; Corrales, L. R., Structure, Dynamics, and Electronic Properties of Lithium Disilicate Melt and Glass. J. Chem. Phys. 2006, 125 (11), 114702.

TOC Graphic

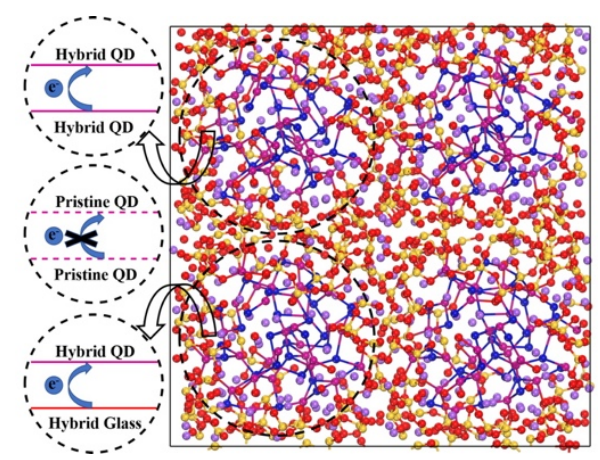

